\title{
Industrial Use Cases of Cyber Physical Systems in EU Projects: Preliminary Study
}

\author{
Rima Al-Ali
}

\begin{abstract}
Smart Cyber-Physical Systems, introduced by Horizon 2020, are the foundation of Industry 4.0 due to the communication between collective intelligent physical devices. Since the usage of sCPS spans over plenty of domains facing variety of challenges, it becomes rather difficult to select an appropriate representative case study for demonstration of approaches developed in research. In this paper, we present a systematic review of sCPS case studies that allows us to identify the domains addressing similar challenges and quantify some of their properties. We group the industrial use cases according to their challenges and map them to the domains.
\end{abstract}

Index Terms - Cyber-Physical Systems, Survey, Demonstrator, Use Case, Application.

\section{INTRODUCTION}

$\mathrm{D}$ EMONSTRATORS, or use cases, are an important tool to illustrate research questions and validate developed ideas, methods, and solutions. Having suitable examples is crucial for demonstration of research results. Moreover, potential variety of domains that the research tackle prove their generality and reusability. Even though the challenges differ across different projects, naturally, they are fixed on a particular one.

Hence, when aiming at development of a "general" method that would be easy to compare with related ones, it is hard to select the right use case for the purpose of presenting and validating. To achieve that, the researchers need to go to the details of each single use case (taken from a selected set) bearing in mind that knowing the domain does not help much in this task. Another option would be to have a predefined use case from an industrial partner, which could limit the method and the solution to be domain-specific.

On the other hand, from the industrial point of view, presenting reusable methods and solutions just in some domains different from theirs makes it harder to match the possibility of applying this particular method on the applications they already have.

Smart Cyber-Physical Systems (sCPS) [10] are interesting for both academic and industrial communities due to the wide range of their applications. The sCPS are introduced in Horizion2020 calls as up-and-coming systems that consider dynamicity within systems involving communication between physical and computational parts known as cyber-physical

The paper is submitted for review on $31 / 5 / 2017$. This research is partially supported by ICT COST Action IC1404 Multi-Paradigm Modelling for CyberPhysical Systems (MPM4CPS) of the H2020 program. systems. In fact, CPSs are considered the foundation of the Industry 4.0 [14], which introduces wireless communication between the embedded devices in manufacturing, aiming at decentralized decision making during a production process.

The current surveys and systematic reviews mostly target CPS architectures (i.e., decomposition into smaller parts and their interconnection) and their applications, which is a well-known method to deal with the increasing complexity of those systems. This includes various aspects, such as Architectural Description Languages (ADL) [16] (called architectural languages (AL) in the paper) and self-adaptation [7], as well as many others [15] [12] [11]. For instance, in [17], the authors are interested in identifying the application domains, challenges, goals, and solutions of architecting CPSs. The systematic study found links between challenges and the suggested solutions for architecting CPSs, which provided a clear idea as to what kind of research could be targeted in the future. In contrast to this, the researchers in [7] headed for finding out application domains and uncertainty types in self-adaptive CPS, and they were able to synthetize patterns from multiple adaptation mechanisms and identify on which layers of the CPS architecture they are applied. As a result of this study, the patterns present an engineering method to be used in designing future self-adaptive CPS.

Regardless of the application domains, in an industrial study [16], the authors raised questions related to the use and the need of ADLs in industry. In this survey, the authors prepared a questionnaire and asked the experts to fill it or made interviews with them. Interestingly, although the ADLs have many domain-specific concepts, the industrial experts consider them very general. However, the study concluded that the needs of industries were not fulfilled by academic ADLs, which highlighted the gap between academic and industrial interests. However, to our best knowledge, no studies involve matching the domains of the collective behavior use cases to their challenges on CORDIS database. Hence, the main goal of this position paper is to present preliminary results on identifying the relations between the demonstrator domains and their challenges. Furthermore, the paper describes an initial grouping of use cases depending on their challenges, which provides other researchers with a wider range of options in choosing an appropriate demonstrator in terms of both the domains and

Rima Al-Ali is a PhD student at Department of Distributed and Dependable Systems, Mathematics and Physics Faculty, Charles University, Prague, Czech Republic (alali@d3s.mff.cuni.cz). 
challenges.

This paper is organized as follows: Section 2 presents the systematic review, while Section 3 presents the results of the study. Section 4 describes our plans for future work and concludes the paper.

\section{THE SYSTEMATIC REVIEW}

To minimize any bias in our research, we decided to perform a systematic review to collect relevant demonstrators for our study. We followed the guidelines of Kitchenham for performing Systematic Literature Reviews in Software Engineering [13]. We briefly summarized the guidelines here to make it easier to follow our steps.

The guidelines start with explaining the need for the systematic review. At the beginning, the researchers have to define the research questions, which the review is expected to answer. In case they would like to know the type and the amount of research, they are heading for systematic mapping review. On the other hand, in case the researchers are experts in a specific area and they seek for more details about the current state of the art, they should take advantage of systematic literature review. The next step is to develop a review protocol, which is a document that includes all systematic review steps and is sent to the reviewers for evaluation. The study has to clarify the source of primary studies, e.g., source: scholar search engines, primary studies: conference papers.

Then, the researchers have to list inclusion and exclusion criteria to be applied on the primary studies. Not only do these criteria help in focusing the results and minimize the number of resulted primary studies, but also they ensure getting the same results if anyone else re-does the review using the same set of primary studies. Moreover, the researchers should define the study procedures, which describe how the selection study is performed. Both study criteria and procedures must have quality assessments to evaluate the sensitivity and the limitations of the study.

From the discovered primary studies, the researchers extract the data needed to address the research questions using data extraction forms. Further, they synthetize the knowledge they are heading for from the data, which is the final result and potentially a subject to dissemination. Of course, the systematic review protocol should include all the information previously acquired in addition to a timetable of the study tasks for the reviewers.

Therefore, following the guidelines in Fig. 1, we start with an overview of our interests in the study.

\section{A. Background}

The requirements for our study have real industrial use cases that target smart Cyber-Physical Systems (sCPS). In this regard, we focused on three basic parts of sCPS: smartness aspect, collective aspect, and physical aspect.

1) Smart Aspect

This part represents the smartness in the system, its ability to perform self-adaption, and make decisions depending on the context.

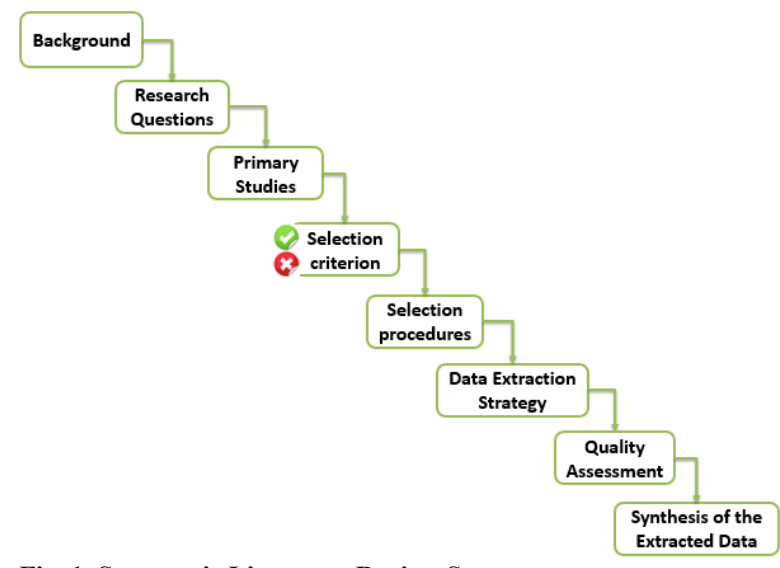

Fig. 1. Systematic Literature Review Steps

2) Collective Aspect

This part represents the collective behavior inside the system where many entities communicate with each other and are involved in forming distributed system behavior, such as in case of Internet of Things (IoT).

3) Physical Aspect

This part considers hardware devices that interact between each other, such as sensors and actuators.

\section{B. Research questions}

The research questions are defined to help in grouping the use cases depending on their challenges. Our study is driven by the following research questions:

RQ1: What are the typical domains for the collective sCPS use cases?

RQ2: What are the principle challenges addressed by the use cases in collective sCPS?

\section{Search for primary studies}

As industrial use cases are our focus in this study, we decided to target the use cases of European projects with Industrial Leadership. The calls that provide such projects (in alignment with our CPS interest) are Information and Communication Technologies (ICT) calls.

Needless to say that the quality of the European projects is high due to all the processes of projects evaluation. In addition, the proposals of the projects have a mandatory requirement to reach high Technology Readiness Level (TRL) [6], which should reach TRL6; this ensures having a working (physical) demonstrator as a requirement for accepting a project proposal. It is worth mentioning that beside the project indicated requirements, we also searched in CORDIS [8] for CPS projects and most of the found projects were accepted under ICT calls, which shows that it the best target for our study.

We considered the projects in the past 10 years as a sample of recent results in this area of research. Therefore, we end up with targeting FP7-ICT and Horizion2020-ICT calls [1] [2] [3] [4] [5] [6]. The calls for FP7 range from 2007 to 2013 and in H2020 from 2014 to 2016 . However, since the projects under H2020 
that started in 2016 are still without results, we have not included them in the review.

Of course, the database is trustworthy since it is from CORDIS on European Commission website, which is the main information source about the European projects.

\section{$D$. Study selection criteria.}

We defined four basic criteria that correspond to the interests of our study. As listed in TABLE I, each of the first three criteria contains a set of representative keywords, which we require to be included in the call and project description. In fact, these keywords are extracted manually from a sample of highly related calls and projects description, and then we filter them to obtain a more representative set.

We include the primary studies (projects) that satisfy all the following constraints (i.e., Inclusion Criteria (IC)):

IC1: Call was made in 2007 - now

IC2: Call title has at least one keyword from at least one of the criteria of the first three ones.

IC3: Call description has one keyword at least from each criteria of the first three ones.

IC4: Project title has at least one keyword from any criteria of the first three ones.

IC5: Project description has at least one keyword from each criteria of the first three ones.

IC6: Project has deliverables, reports or a demonstrator description.

IC7: Challenges of the use cases target collective behavior.

IC8: Use cases have more than two interacting entities, which represent the collective behavior (e.g., many robots work together, many robots interact with humans)

We exclude the projects (i.e., Exclusion Criteria (EC)) according to the following exclusion criteria:

EC1: Call started 2016-2017 since it is without results yet (i.e., no periodic report)

EC2: Call description contains only keywords in context different from one of the three first criteria (e.g., collaboration between researchers).

EC3: Project description contains only keywords in context different from one of the three first criteria (e.g., collaboration between researchers).

EC4: Project does not have working website.

EC5: Project has no deliverables, reports nor a demonstrator description.

EC6: Use case challenges are unrelated to collective behavior. EC7: Use cases represent a collective knowledge exchange between people (e.g., social networks, software for rating places, roadmaps, and platforms).

EC8: Use cases have one-to-one or one-to-many relation between robots and human (e.g., wearables, tour guide robot).

\section{E. Study selection procedures.}

The selection procedures are presented in four basic parts: primary studies, calls, projects, and use cases.
TABLE I

SELECTION CRITERIA

\begin{tabular}{|c|c|c|c|}
\hline $\begin{array}{l}\text { Criteria } 1 \\
\text { Smart aspect }\end{array}$ & $\begin{array}{l}\text { Criteria } 2 \\
\text { Collective aspect }\end{array}$ & $\begin{array}{l}\text { Criteria } 3 \\
\text { Physical aspect }\end{array}$ & $\begin{array}{l}\text { Criteria } 4 \\
\text { Entities }\end{array}$ \\
\hline $\begin{array}{l}\operatorname{smart}(*) \\
\text { intelligen }(*) \\
\operatorname{adapt}(*) \\
\operatorname{autonom}(*) \\
\operatorname{aware}(*)\end{array}$ & $\begin{array}{l}\text { distribut(*) } \\
\text { de(-)central }(*) \\
\text { co(-)operat }(*) \\
\text { communicat }(*) \\
\text { collaborat }(*) \\
\text { connective }(*) \\
\text { emergent } \\
\text { swarm } \\
\text { collective }\end{array}$ & $\begin{array}{l}\text { physical } \\
\text { CPS } \\
\text { Internet of Things } \\
\text { IoT } \\
\text { embedded } \\
\text { device } \\
\text { hardware } \\
\text { robot }\end{array}$ & $\#>2$ \\
\hline
\end{tabular}

*) means zero or several letters

$(-)$ means it is possible to contain hyphen in the word

\# means number of entities or nodes that interact in use case

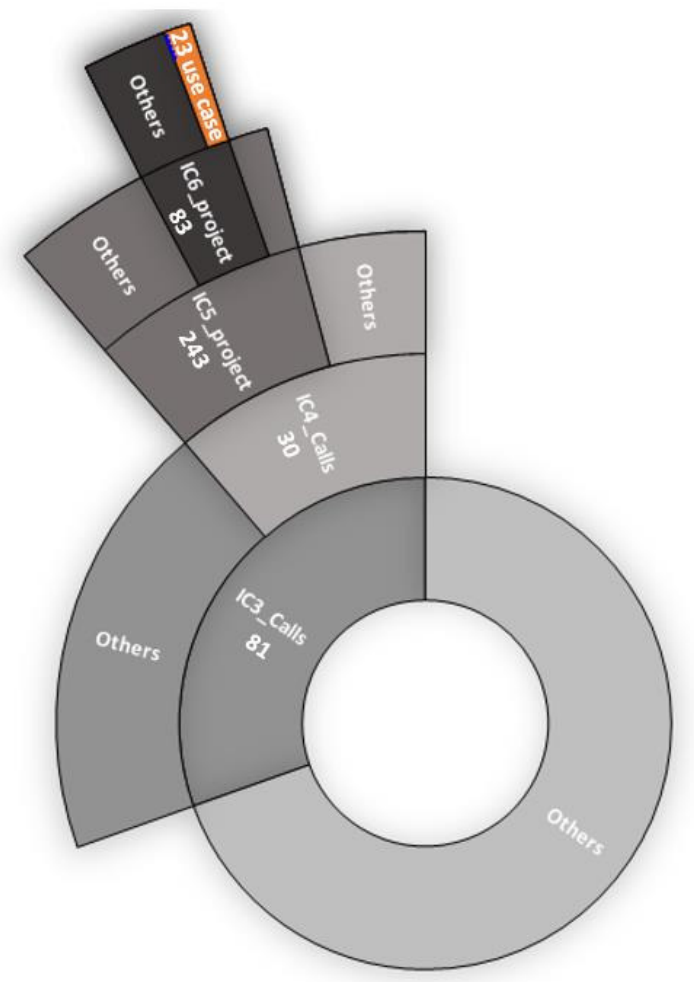

Fig. 2. The Selection of Use Cases - The Final Set in Orange

The first part is related to the study foundation, and the procedure follows the guidelines in [13]. Therefore, we started with: (1) describing the background of the research interest, followed by (2) defining the research questions. Afterwards, (3) we selected the calls of Information and Communication Technologies (ICT), which are FP7 and Horizion2020 [9] calls from 2007 to 2016 as a target [IC1]. Next, (4) we defined four criteria out of which three are the sets of keywords (i.e. see TABLE I).

The second part is related to selection of ICT Calls, where (5) we looked up for any of the keywords in the title of the calls [IC2], (6) excluding the 2016-2017 calls since the corresponding projects do not have results on the website yet [EC1]. Afterwards, (7) we searched in the resulted calls description for at least one keyword from each criteria [IC3] [EC2]. Then, from the final filtered calls, (8) we created a report containing the all the projects. 
The third part is related to project selection, which starts with (9) looking up for any of the keywords in the title of the projects [IC4]. Then, (10) in the resulted projects, we looked up for each project description that contains at least one keyword from each criteria [IC5] [EC3]. Further, (11) we filtered out the projects that are without a working website [EC4]. In case that the website in CORDIS does not work, we looked up for the website using the "(project title) + project" as the search string in Google. Then, (12) we selected the projects that had documentation for their demonstrators as a description in the website, deliverables, or reports [IC6] [EC5]. Finally, (13) from the final set of projects, we created a list of all corresponding use cases.

The fourth and the last part is related to selecting the use cases. Here, (14) we select the use cases with challenges that target collective behavior [IC7][EC6][EC7]. After that, (15) we selected the use cases that are designed for more than two nodes [IC8][EC8].

\section{F. Study quality assessment checklists and procedures.}

This part requires having an evaluation from reviewers, which we still miss as this work is still in progress. However, we plan to have the study reviewed by at least two experts. Nevertheless, in the future work section below, we discuss the sensitivity and the quality of our study that is affected by many points, such as the selection of the primary studies, the selection of the keywords sets, the evaluation of collective challenges, and the evaluation of the number of nodes.

\section{G.Data extraction strategy}

We have used pre-defined extraction forms (i.e. see TABLE II, TABLE III, TABLE IV). Each table contains basic information that is needed to apply the selection procedures. Therefore, we defined a form for all calls, which required the title, the description of the call, and the criteria $1|2| 3$ fields that hold the extracted data from the project descriptions. Similarly, the second form is related to the project data, which includes the title, the description, website availability, and the criteria $1|2| 3$ fields. The last form contains use cases information, which consists of use case description, its domain, its challenges, the number of its nodes, and its documentation availability. Finally, the targeted data to address our research questions is in the "Use Case Domain" and "Use Case Challenges". The former one is related the RQ1, while the latter is related to RQ2.

\section{RESULTS}

After applying the method on all H2020 and FP7 calls, the results of research questions are extracted and further analyzed from the point of view of both domain and challenges fields. In the following sections, we present our results.

\section{A. General Statistics}

Fig. 2 describes the numbers of each selection procedures, which starts with total number 266 of the presented calls in both FP7 and H2020. After applying the selection procedure to calls using steps $1-8$, the number of selected calls decreases to 30 ,
TABLE II

DATA EXTRACTION FORM FOR CALLS

\begin{tabular}{ll}
\hline \hline Data Item - Call & \\
\hline Call ID: & Call Title: \\
Call Description: & Criteria2: \\
Criteria1: & \\
Criteria3: & \\
\hline \hline
\end{tabular}

"Call Description" is the text in ICT work program.

TABLE III

DATA EXTRACTION FORMS FOR PROJECTS

\begin{tabular}{ll}
\hline \hline Data Item - Project & \\
\hline Call ID: & Project ID: \\
Project Name: & Project Title: \\
Description: & \\
Criteria1: & Criteria2: \\
Criteria3: & \\
Website: & \\
\hline \hline
\end{tabular}

"Project Description" is the text in CORDIS website.

TABLE IV

DATA EXTRACTION FORM FOR USE CASES

\begin{tabular}{ll}
\hline \hline Data Item - Use Cases & \\
\hline Project ID: & Use Case ID: \\
Domain: & Use Case Description: \\
Demonstrator: & Challenges: \\
Criteria4: & \\
Demonstrator Description (yes/no) & Deliverables and reports (yes/no) \\
\hline \hline
\end{tabular}

"Use Case Description" is the description in the project website or in the deliverables. "Demonstrator" is an abstract of the use case description. "Challenges" is the abstract of use case challenges.

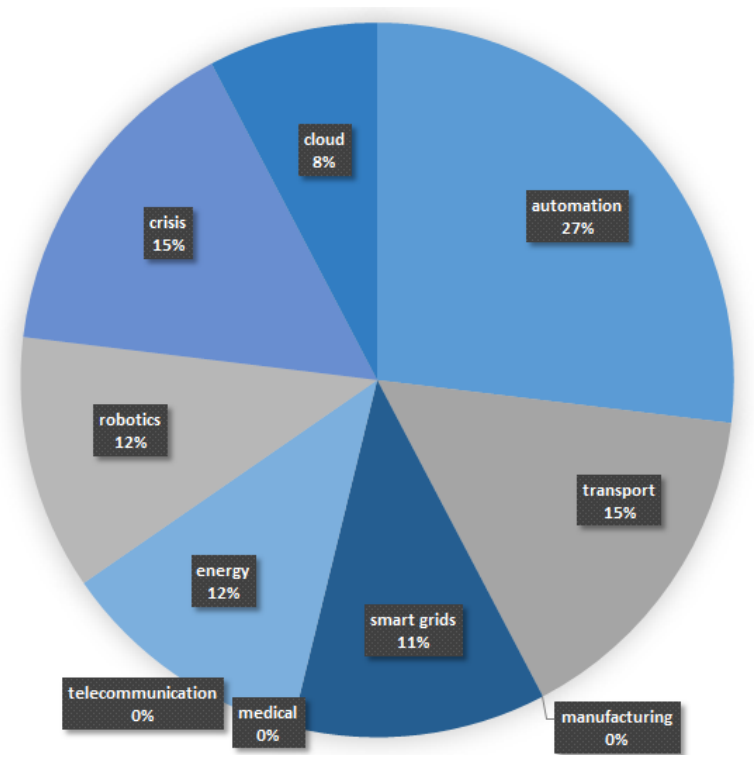

Fig. 3. Domains Percentage of the Selected Use Cases

which corresponds to 348 projects. Then, after applying steps 9-13, the number of the selected projects went down to 83, which corresponds to 96 use cases. Finally, after applying steps $14-15$, the final number of selected use cases is 23 .

\section{B. Use Cases Domains (RQ1)}

The domains of the selected use cases after applying selection procedures are: clouds, crisis, robotics, telecommunication, energy, medical, smart grids, manufacturing, automation, and transport (i.e. see Fig. 3). There is some overlap between those domains, but we related each use case to just one domain except for three use cases that stated multiple domains explicitly in 
TABLE V

GROUPING CHALLENGES

\begin{tabular}{|c|c|c|c|c|c|c|}
\hline Safety & Awareness & Uncertainty & Collective goal & Communication & Resources & Planning \\
\hline $\begin{array}{l}\text { overcome failure of } \\
\text { individual }\end{array}$ & $\begin{array}{l}\text { environment- } \\
\text { aware }\end{array}$ & $\begin{array}{l}\text { uncertainty in sensors } \\
\text { data }\end{array}$ & $\begin{array}{l}\text { reach inaccessible } \\
\text { /wide areas }\end{array}$ & $\begin{array}{l}\text { short range } \\
\text { communication }\end{array}$ & sharing resources & \\
\hline unexpected failure & dynamicity & unpredictable dynamics & achieve shared goal & limited communication & energy demand & \\
\hline recover failure & situation-aware & & team goal & network problems & cost & \\
\hline safety & & & & delays & load distribution & \\
\hline \multirow[t]{5}{*}{ human safety } & & & & & limited time & \\
\hline & & & & & energy waste & \\
\hline & & & & & energy efficiency & \\
\hline & & & & & performance & \\
\hline & & & & & traffic load & \\
\hline
\end{tabular}

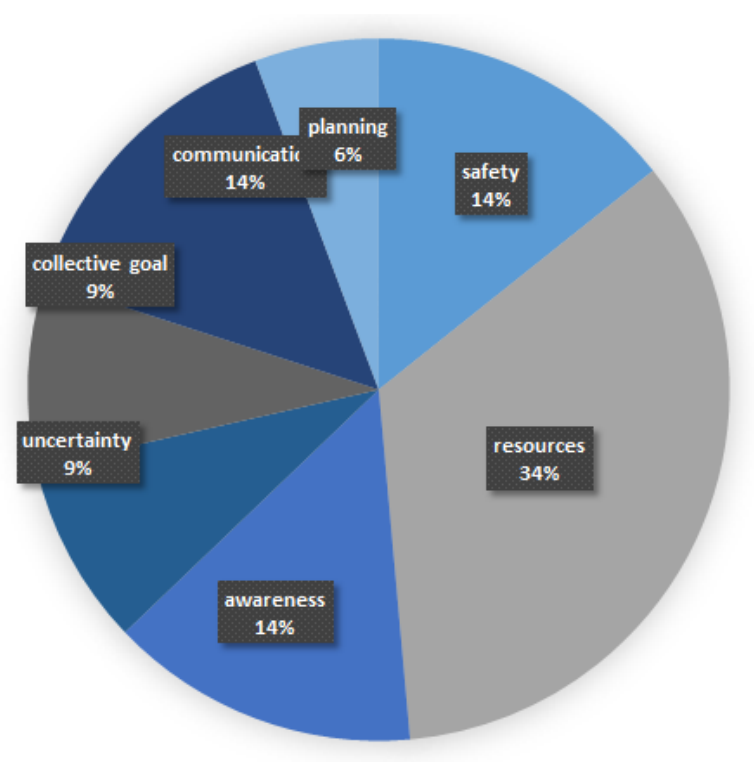

Fig. 5. Challenges Percentage in the Selected Use Cases

their descriptions (the use cases are pilots of a smart city scenario).

The automation use cases cover various views of automation, such as building automation, railways and industry automation (e.g., home management, decentralized management of manufacturing entities), while in case of transport, the use cases target planning, and e-charging and sharing parking places. In case of crisis, the usual use case is having a team of collaborating robots and humans that aim at saving lives. Despite the fact that most of the robotics use cases focus on learning and better interaction with human, some of them involve groups of robots that aim at achieving a common goal collectively.

In case of smart grids and energy, both domains have the energy management scenario. For clouds, this involves performance and distributing load.

Most of the use cases in telecommunication were concerned with mobile applications for exchanging knowledge between people (e.g., social networking). As to the medical use cases, they focused on monitoring and developing specialized robots that helped elderly people or people in need (e.g., in the form of a wearable to keep the balance during walking). Regarding manufacturing, this domain contains developing and building the hardware more than using it in a collective use case.

As illustrated by the chart in Fig. 3, the answer of the first research question "RQ1: What are the typical domains for the

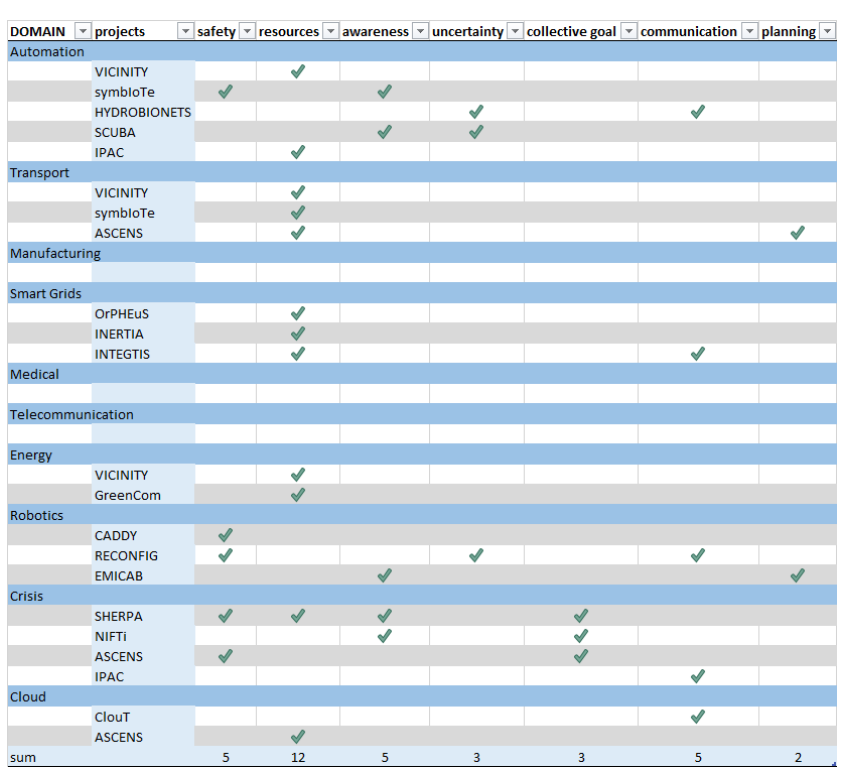

Fig. 4. Mapping the Challenges to Use Cases Domain

smart collective CPS use cases?" is "automation", then "transport", and "crisis".

\section{Use Cases Challenges (RQ2).}

The challenges of the selected use cases after applying selection procedures are grouped into the following representative ones (TABLE V): safety, resources, awareness, uncertainty, collective goal, communication, monitoring, and planning (Fig. 5).

The resource group, which is the biggest one, targets any kind of resources such as time, energy, and cost. The safety group includes every challenge that corresponds to safety of the system or its entities including humans, in addition to safe recovery from failures. Further, awareness group targets a collective awareness of the environment and the context that surrounds the system entities for a better performance. Another important challenge group is communication; this group targets any problem that could happen with the communication in the system stemming from its limitations, failures or even a lack of it.

Even though collective goal groups are less addressed in our selected use cases, they form a significant part in CPS. More specifically, the system entities in CPS tend to be grouped in a decentralized way to achieve common goals that they are not able to reach alone or it is more costly. Although uncertainty exists everywhere inside and outside of a sCPS system, it is 
explicitly targeted here in data uncertainty and situation unpredictability. It is worth mentioning that uncertainty also can be caused by delays, problems in networking, unexpected failures, and unexpected situations, which means it is involved in more than one group. Since we tried to have a reasonable representation for it, we introduced a separate group for it.

As illustrated in Fig. 4, the answer of the second research question "RQ2: What are the principle challenges addressed by the use case?" is resources, then safety, communication, and awareness.

\section{D.Mapping Challenges to Domains}

In Fig. 4, we matched the representative challenges of the selected use case to their domains. As a result, we notice that resources and communication as a challenges target most of the domains, Moreover, awareness as well as safety are very important to automation and crisis in addition to robotics domains since it is required to have a high level of realization to the surroundings due to its criticality and cost.

Surprisingly, the only domain where the collective goal is addressed is crisis, while planning gets the interest of transport and robotics domains; uncertainty are considered in automation and robotics.

To sum up, the resources and communication cover most of the domains, while there is a lack in addressing other challenges in transport, cloud, energy, and smart grids. Also, manufacturing, telecommunication, and medical domains are not represented well by industrial use cases of collective sCPS.

\section{CONCLUSION AND FUTURE WORK}

Smart CPSs are spreading around with their possible applications in industries as well as in academic research. Therefore, we need a way to find out what challenges correspond to which domains in industrial use cases. For this reason, we did a systematic mapping review to find the domains and the challenges that industry targets nowadays. The primary studies that we used are EU projects from the ICT calls since 2007 until now.

The results of the study showed that the most targeted domain is automation and the most addressed challenge is resources. Moreover, domains such as transport, cloud, energy, and smart grids lack variety of challenges, while many other domains do not target any collective challenge. Those include telecommunication, medical and manufacturing domains.

As future work, we plan to validate the results and extend the study to contain academic use cases in the aim of comparing the difference in interests between industrial-based and academicbased use cases, regarding domains and challenges. Furthermore, we plan to synthetize a small set of representative use cases for the presented challenges to be used in case of presenting a general method instead of using a domain specific use case.

\section{V.APPENDIX}

In this appendix, we present in TABLE VI the list of project that contains the final selected set of use cases.

\section{ACKNOWLEDGMENT}

This paper is partially supported by ICT COST Action IC1404 Multi-Paradigm Modelling for Cyber-Physical Systems (MPM4CPS) of the H2020 program. Additionally, I would like to express my thanks of gratitude to Tomáš Bureš, Jan Kofroň, and Lubomír Bulej, who helped me during my work.

TABLE VI

Projects With Selected USE CASES

\begin{tabular}{ll}
\hline \hline $\begin{array}{l}\text { Project } \\
\text { Name }\end{array}$ & Project Title \\
\hline VICINITY & $\begin{array}{l}\text { Open virtual neighbourhood network to connect } \\
\text { intelligent buildings and smart objects } \\
\end{array}$ \\
& Website: http://vicinity2020.eu/vicinity/
\end{tabular}

symbIo Symbiosis of smart objects across IoT environments Website: https://www.symbiote-h2020.eu/

CADDY Cognitive autonomous diving buddy Website: http://caddy-fp7.eu/

OrPHEuS OPtimising Hybrid Energy grids for smart citieS Website: http://www.orpheus-project.eu/

ClouT ClouT: Cloud of Things for empowering the citizen clout in smart cities Website: http://clout-project.eu/

SHERPA Smart collaboration between Humans and ground-aErial Robots for imProving rescuing activities in Alpine environments

Website: http://www.sherpa-project.eu/sherpa/

RECONFIG Cognitive, Decentralized Coordination of Heterogeneous Multi-Robot Systems via Reconfigurable Task Planning Website: http://www.reconfig.eu/

HYDROBIO Autonomous Control of Large-scale Water Treatment NETS Plants based on Self-Organized Wireless BioMEM Sensor and Actuator Networks

Website: http://www.hydrobionets.eu/

SCUBA SCUBA - Self-organising, Cooperative, and robUst $\backslash n$ Building Automation

Website: http://www.aws.cit.ie/scuba/

GreenCom MyGrid; Energy Efficient and Interoperable $\backslash n$ Smart Energy Systems for Local Communities Website: http://www.greencom-project.eu/

INERTIA Integrating Active, Flexible and Responsive Tertiary Prosumers into a Smart Distribution Grid Website: http://www.inertia-project.eu/inertia/

EMICAB Embodied Motion Intelligence for Cognitive, Autonomous Robots

Website: http://www.emicab.eu/

NIFTi Natural human-robot cooperation in dynamic environments Website: http://www.nifti.eu/

INTEGRIS INTelligent Electrical Grid Sensor communications Website: http://fp7integris.eu/index.php

ASCENS Autonomic Service-Component Ensembles Website: http://www.ascens-ist.eu/

IPAC Integrated Platform for Autonomic Computing Website: http://ipac.di.uoa.gr/ 


\section{REFERENCES}

[1] European Commission. ICT work programme 2007/2008, FP7 EU projects. [Online]. Available: http://ec.europa.eu/research/participants/ data/ref/fp7/88465/c-wp-200801_en.pdf, 2007

[2] European Commission. ICT work programme 2009/2010, FP7 EU projects. [Online]. Available: http://cordis.europa.eu/pub/fp7/ict/docs/ ict-wp-2009-10_en.pdf, 2009

[3] European Commission. ICT work programme 2011/2012, FP7 EU projects. [Online]. Available: http://cordis.europa.eu/fp7/ict/ components/documents/ict-wp-2011-12-en.pdf, 2011.

[4] European Commission. ICT work programme 2013, FP7 EU projects. [Online]. Available: https://cordis.europa.eu/fp7/ict/docs/ict-wp201310-7-2013-with-cover-issn.pdf, 2013.

[5] European Commission. ICT work programme 2014/2015, H2020 EU projects. [Online]. Available: https://ec.europa.eu/research/ participants/data/ref/h2020/wp/2014_2015/main/h2020-wp1415-leitict_en.pdf, 2014.

[6] European Commission. ICT work programme 2016/2017, H2020 EU projects. [Online]. Available: http://ec.europa.eu/research/participants/ data/ref/h2020/wp/2016_2017/main/h2020-wp1617-leit-ict_en.pdf, 2016.

[7] A. Musil, et al., "Patterns for Self-Adaptation in Cyber-Physical Systems Multi-Disciplinary Engineering for Cyber-Physical Production Systems," Data Models and Software Solutions for Handling Complex Engineering Projects, Springer International Publishing, pp. 331-368, May 2017, doi: 10.1007/978-3-319-56345-9_13.

[8] European Commission. Cordis. [Online]. Available: http://cordis. europa.eu/home_en.html
[9] European Commission. Horizon 2020. [Online]. Available: https://ec.europa.eu/programmes/horizon2020/

[10] European Commission. Smart cyber-physical systems, Horizon 2020. [Online]. Available: https://ec.europa.eu/programmes/horizon2020/en/ h2020-section/smart-cyber-physical-systems

[11] D. Dac Hoang, H. Paik, and C. Kim, "Service-oriented middleware architectures for cyber-physical systems," IJCSNS International Journal of Computer Science and Network Security, vol.12, no. 1, January, 2012.

[12] P. Derler, E. A. Lee, and A. Sangiovanni Vincentelli, "Modeling Cyber Physical Systems," Proceedings of the IEEE, vol.100, no.1, pp. 13-28, January 2012, , doi: 0.1109/JPROC.2011.2160929.

[13] B. Kitchenham and S. Charters, "Guidelines for performing Systematic Literature Reviews in Software Engineering," Technical Report, EBSE-2007.

[14] Y. Lu, "Industry 4.0: A Survey on Technologies, Applications and Open Research Issues," Journal of Industrial Information Integration, vol. 6, pp. 1-10, June 2017, doi: 10.1016/j.jii.2017.04.005.

[15] C. A. Macana, N. Quijano, and E. Mojica-Nava, "A survey on Cyber Physical Energy Systems and their applications on smart grids," In 2011 IEEE PES Conference on Innovative Smart Grid Technologies Latin America (ISGT LA), pp. 1-7, October 2011, doi: 10.1109/ISGTLA.2011.6083194.

[16] I. Malavolta, et al., "What Industry Needs from Architectural Languages: A Survey," IEEE Transactions on Software Engineering, vol. 39, no. 6, pp.869-891, June 2013, doi: 10.1109/TSE.2012.74.

[17] I. Malavolta, H. Muccini, and M. Sharaf, "A preliminary study on architecting cyber-physical systems," In Proceedings of the 2015 European Conference on Software Architecture Workshops, ECSAW '15, pp. 1-6, New York, NY, USA, 2015, doi: 10.1145/ 2797433.2797453. 